Review began 11/01/2021 Review ended 11/06/2021 Published 11/10/2021

๑) Copyright 2021

Hussain et al. This is an open access article distributed under the terms of the Creative Commons Attribution License CC-BY 4.0., which permits unrestricted use, distribution, and reproduction in any medium, provided the original author and source are credited.

\section{Safety Evaluation of an Expedited Omalizumab Home Self-Administration Pathway}

\author{
Zobia Hussain $^{1}$, Lisa Devlin ${ }^{2}$ \\ Belfast, GBR \\ Corresponding author: Lisa Devlin, lisa.devlin@belfasttrust.hscni.net
}

1. Emergency Medicine, Royal Victoria Hospital, Belfast, GBR 2. Allergy and Immunology, Royal Victoria Hospital,

\section{Abstract \\ Introduction}

The Northern Ireland Regional Immunology Service (NIRIS) has developed an expedited omalizumab home self-administration pathway to reduce face-to-face clinic attendance during the coronavirus disease 2019 (COVID-19) pandemic. This audit evaluates the safety of this pathway with a particular focus on anaphylaxis.

\section{Objectives}

This study aimed to retrospectively audit the records of 39 patients undertaking expedited home selfadministration at NIRIS for complications, particularly emergency department attendance for anaphylaxis. The target was for $100 \%$ of patients to complete a six-month course without experiencing anaphylaxis related to omalizumab administration.

\section{Materials and methods}

A total of 39 records of patients who underwent expedited omalizumab self-administration were audited by a single reviewer. They were prospectively collected between March 2020 and August 2021. Clinical data were collected from the Northern Ireland Electronic Care Record (NIECR).

\section{Results}

Hundred percent of patients were in the process of completing or had completed a six-dose course without anaphylaxis. During the course of omalizumab, $7.6 \%$ of patients attended the emergency department. Zero percent of patients have experienced anaphylaxis triggered by omalizumab. The target of $100 \%$ patients completing the expedited pathway without omalizumab-related anaphylaxis was met.

\section{Conclusion}

Home self-administration of omalizumab is preferred by patients and clinicians for reducing expense, travel, and unnecessary clinical contact during the COVID-19 pandemic. An expedited omalizumab home selfadministration training pathway appears to be safe in a population of Northern Irish patients with chronic spontaneous urticaria (CSU). More research is needed to determine whether the expedited pathway should become the standard of care post-pandemic

Categories: Allergy/Immunology

Keywords: covid-19, anaphylaxis, patient safety, self-administration, service provision, chronic spontaneous urticaria, omalizumab, immunology

\section{Introduction}

Omalizumab (Xolair@) is an anti-IgE monoclonal antibody used as adjunctive therapy in the management of chronic spontaneous urticaria (CSU) and moderate-to-severe allergic asthma [1]. As a CSU treatment, it is administered as a subcutaneous injection once every four weeks for a course of six treatments. These treatments were previously only licensed for administration by a healthcare professional under medical supervision due to a perceived risk of anaphylaxis, although the documented risk in the literature was low $(0.1 \%)[2]$.

The coronavirus disease 2019 (COVID-19) pandemic significantly disrupted healthcare, and services were required to reduce face-to-face attendances in order to decrease transmission of the virus. The British Society of Allergy and Clinical Immunology (BSACI) published recommendations relating to home therapy included expediting home self-administration pathways and extra-license usage of home selfadministration in patients with a history of anaphylaxis which had a clear trigger unrelated to omalizumab [3]. The Northern Ireland Regional Immunology Service has developed an omalizumab home 


\section{Cureus}

self-administration pathway in which patients receive four doses under medical supervision with training on how to self-administer, followed by home self-administration for the remainder of the course. Weighing up the low risk of anaphylaxis against the benefit of reducing footfall during the pandemic, it was determined that new patients may receive one treatment and training under medical supervision followed by home self-administration instead of the standard four doses. This safety audit investigates the safety of the expedited omalizumab home self-administration pathway.

\section{Materials And Methods}

This is a retrospective audit assessing the total number of patients who underwent expedited omalizumab self-administration between March 2020 and August 2021 (N=39). Patients were identified by the clinical coordinator from a departmental spreadsheet. Clinical data were collected from the Northern Ireland Electronic Care Record (NIECR). Data were put into and analysed using Microsoft Excel by a medical student. The objective of this study was to identify the incidence of anaphylaxis in patients on the expedited omalizumab home self-administration pathway. The standardised procedure followed in omalizumab home self-administration training is mentioned in Appendices. The audit standards are mentioned in Table 1 below.

\section{Audit criteria}

Patients receiving expedited omalizumab home self-administration therapy commence a six-dose course without experiencing omalizumab-related anaphylaxis (criterion 1)

\section{Target}

(\%)

Exceptions Source of evidence

BSACl: modifications for

Nil adult allergy services during the COVID-19 pandemic

TABLE 1: Omalizumab home self-administration safety audit standards

BSACI: British Society of Allergy and Clinical Immunology; COVID-19: coronavirus disease 2019

\section{Results}

The results related to criterion 1 are mentioned in Table 2 below. Hundred percent (39/39) of patients were in the process of completing or had completed a six-dose course without complications. Zero out of 39 (0\%) attended experienced anaphylaxis triggered by omalizumab. The target of $100 \%$ patients completing the expedited pathway without omalizumab-related anaphylaxis was met.

\begin{tabular}{|l|l|l|l|l|}
\hline Target & Total patients & Patients meeting criterion $\mathbf{1}$ & Percentage patients meeting target & Was the target met? \\
\hline $100 \%$ & 39 & 39 & $100 \%$ & Yes \\
\hline
\end{tabular}

TABLE 2: Percentage of patients meeting audit target

Criterion 1 - patients receiving expedited omalizumab home self-administration therapy commence a six-dose course without experiencing anaphylaxis.

Three out of 39 (7.6\%) attended the emergency department during the course of their treatment with omalizumab. One of these patients attended because of visual disturbances unrelated to omalizumab. The second was receiving omalizumab for eight weeks for a year prior to attending the emergency department with a widespread rash, no associated airway compromise or hypotension; it was determined not to be anaphylaxis and the patient continued their course of omalizumab without issue. The third patient had already received two doses of omalizumab without issue, once under clinical supervision and once selfadministered at home. Approximately two weeks after self-administering the third dose, the patient experienced a flare of their urticaria with lip swelling and throat tightening. This patient was treated with antihistamines, oral corticosteroids, and discharged; they received a subsequent dose of omalizumab without issue. The flare which occurred after the third dose suggests treatment failure. We can conclude however that this incident was not related to anaphylaxis. Patients with omalizumab-related anaphylaxis would present with hypotension in addition to airway obstruction, it is also extremely unlikely to occur after receiving a third dose when the previous two doses were well-tolerated. Hence, due to the timing and nature of the symptoms, it was determined to be a flare of CSU rather than anaphylaxis.

\section{Discussion}

The requirement to administer omalizumab only under medical supervision causes significant disruption to 
patients' daily activities, particularly for patients commuting long distances to a regional centre for each treatment [4]. In late 2018, omalizumab was approved for home self-administration in patients without a history of anaphylaxis, which bypasses some of these issues. Timmermann and Mailänder conducted a survey of patients being treated with omalizumab in 2020 and found that the majority of patients were in favour of home self-administration, with time efficiency being the primary advantage identified [4]. In simulations carried out by Shaker et al., there was a significant difference in cost-effectiveness of in-clinic omalizumab administration compared to home self-administration, with the latter being more costeffective [5]. These findings are well supported in the literature [2,6-8]. Ninety-three percent of patients favour home self-administration which further supports the benefits of the expedited pathway [9].

While previous debate hinged primarily on the perceived risk of anaphylaxis, the COVID-19 pandemic pushed the issue of reducing the clinic burden to the forefront. Telehealth became increasingly important due to social distancing regulations. A 2020 systematic review found that virtual clinics improved the provision of healthcare and reduced the transmission of the virus during the outbreak [10]. Studies found a mixed patient response to pandemic telehealth measures [11-13] but found robust evidence concerning safety in light of the pandemic [14-16].

In light of evidence suggesting that face-to-face clinics could be safely cut down without compromising clinical care, BSACI published recommendations on strategies to minimise clinic footfall. Recommendations included deferring new treatment courses of omalizumab until the pandemic subsided, increasing the interval between doses, or discontinuing the treatment in patients partway through a course. Previously strict guidelines were loosened to allow extra-license usage of home self-administration in patients with a history of anaphylaxis which had a clear trigger unrelated to omalizumab or in shortening the pathway [3]. The effects of the pandemic and these guidelines in the real world were investigated by Krishna et al. [17]. It was found that $86-92 \%$ of immunology services continued routine appointments including new referrals but most of these consultations were telehealth or virtual, as there was a significant reduction in face-to-face consultations amongst the 99 immunology and allergy services participating in the study. The vast majority of services changed immunoglobulin administration regimens and reduced sublingual and subcutaneous injection immunotherapy among other therapeutic procedures. Adverse outcomes relating to these changes primarily centred around the immunoglobulin replacement therapy, although some centres reported exacerbations of CSU relating to missed omalizumab doses [17]. One flaw of these reports is inconsistent coding of anaphylaxis and urticarial symptoms; it is possible that the incidents of urticaria may have been misdiagnosed as anaphylaxis, falsely raising the rate of this complication. The Northern Ireland Regional Immunology Service (NIRIS) did not report any such adverse events owing in large part to the timely expedited home self-administration pathway, which successfully allowed continuing treatment alongside reducing the risk of COVID-19 spread. Indeed, from the reports in the literature on similar practices elsewhere, it appears that the risk of anaphylaxis may be overblown and that the benefits of empowering patients to take charge of their care may continue to benefit them in post-pandemic [17].

\section{Limitations}

The primary limitations of this study centre around the small sample size of patients investigated (N=39). This audit should be repeated in six to 12 months to validate previous results and investigate temporal trends. It is possible that a degree of selection bias may be present in those patients who choose to undergo home self-administration training may be less likely to experience anaphylaxis; however, anaphylaxis is unrelated to lifestyle factors so this bias is unlikely. The population of Northern Irish CSU patients may have different characteristics from the larger population of CSU patients. More research is needed to conclusively determine the safety of this approach.

\section{Conclusions}

Although omalizumab home self-administration is considered controversial due to a perceived risk of anaphylaxis, the results of this audit suggest that allowing Northern Irish patients to complete the pathway after a single supervised treatment is safe. There was a clinical need for an expedited self-administration pathway during the COVID-19 pandemic, and the expedited pathway was an appropriate measure to reduce face-to-face interaction, hence reducing transmission of the virus. It is plausible that this expedited pathway could be continued after pandemic restrictions are lifted, which could be beneficial to both patients and clinicians by increasing time efficiency. More research is needed before generalising these findings to other patient populations.

\section{Appendices}

\section{Standard 4.2.1 training procedure for self-administration of omalizumab}

4.2.1.a: Introduction

Selected patients who are established on omalizumab for chronic spontaneous urticaria will be given the option to be trained for self-administration within the home setting. 


\section{Cureus}

This document outlines the procedure to be followed in selecting and training patients.

4.2.1.c: Related Documents

1. Standard 4.2.2 Omalizumab (Xolair) for the treatment of Chronic Spontaneous Urticaria

2. Sign 153 - British guideline on the management of asthma, September 2016

4.2.1.d: Procedure

(A) Selection of patients for self-administration of omalizumab

1. All patients must have had four doses of omalizumab administered in the hospital setting without any adverse effect, such as urticaria; angioedema; respiratory distress - wheeze, dyspnoea,

tachypnoea; vomiting, diarrhoea, severe abdominal discomfort.

2. Patients must consent to train

3. All patients with asthma/COPD should be discussed with a member of the medical team before training for self-administering omalizumab.

4. Patients with poorly controlled or severe asthma will be excluded from self-administering omalizumab: (a) in adults: FEV1 < 70\% of the predicted value after adequate pharmacologic treatment; (b) patients on high dose therapies or continuous/frequent use of oral corticosteroids (see SIGN 154 British guidelines on the management of asthma)

(B) Consent for home administration of omalizumab - agreement form (Figure 1)

\section{Home Therapy; self-administration of subcutaneous omalizumab}

I would like to attend the Home Therapy Training Course for selfadministration of subcutaneous Omalizumab at home.

I understand this will involve attending a training programme and I intend to give my own injection if I feel confident to do so.

I have read the information concerning subcutaneous infusions and I agree to carry out the infusions as I have been trained.

I agree to visit my hospital for three monthly follow-up appointments and be available for an annual assessment/retraining as required.

I agree to maintain my home supply of Omalizumab as requested.

I understand that I should comply with these stipulations if I wish to continue administering Omalizumab at home.

\section{Patient \\ Signature Name (Print) \\ Date \\ Telephone \\ number}

\author{
Assistant \\ Signature \\ Name (Print) \\ Date \\ Telephone \\ number
}

FIGURE 1: Omalizumab home administration consent form

(C) Training procedure for injection of omalizumab

1. Equipment required - clean tray or tabletop (wiped down with steret); alcohol skin wipes; plaster; sharps box; waste-paper bin; omalizumab injection

2. Method - (i) wash and dry hands; (ii) remove the syringe from the refrigerator 30 minutes before 


\section{Cureus}

administration to allow it to reach room temperature. If not administered the syringe can be returned to the fridge ONCE only. However, the time out of the fridge must not exceed four hours; (iii) assemble equipment in a clean area; (iv) clean area of skin with an alcohol wipe; (v) holding the syringe with the needle pointing upwards, carefully pull off the needle cap from the syringe and discard it. Do not touch the exposed needle. Then, gently tap the syringe with your finger until the air bubble rises to the top of the syringe. Slowly push the plunger up to force the air bubble out of the syringe without inadvertently expelling the solution. (vi) Gently pinch the skin of your upper thigh or abdomen and insert the needle; (vii) holding onto the finger flange, slowly depress the plunger as far as it will go. If any solution leaks from the injection site, insert the needle further; (viii) keeping the plunger fully depressed, carefully lift the needle straight out from the injection site; (ix) slowly release the plunger and allow the needle guard to automatically cover the exposed needle; (x) dispose of the syringe in the sharps container

(D) Training record for self-administration of omalizumab (Figure 2; see Table 3 for urticaria activity score $\{\mathrm{UAS}\})$. 


\section{Cureus}

TRAINING RECORD FOR SELF ADMINISTRA TION OF OMALIZUMAB

Patient addressograph

Name of injection partner being trained (if applicable)

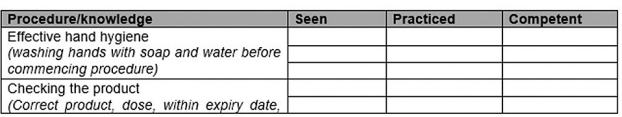

dose, within expiry

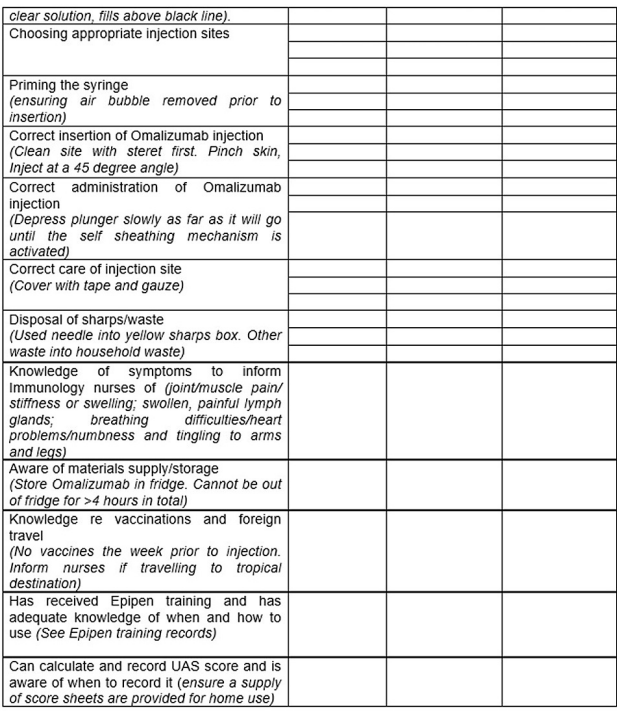

aware of when to record it (ensure a suppoy

(Initials of trainer and date entered where appropriate in above table)

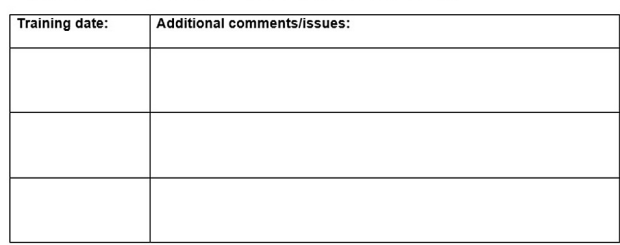

Complete when all criteria have been assessed at competent level:

Competent at injecting omalizumab independently YES

Comments:

Allergy Nurse Specialist (Print)

Signature Date

I confirm I have received training on how to administer my omalizumab
injections independently at home and am confident and competent in

injections independenty at home and am confident and competent

Patient Name (Print)

Signature

Date

FIGURE 2: Omalizumab home self-administration training record 


\section{Cureus}

\begin{tabular}{|c|c|c|c|}
\hline $\begin{array}{l}\text { Itch severity } \\
\text { score }\end{array}$ & Itch severity (once every $24 \mathrm{~h}$ ) & $\begin{array}{l}\text { Hives severity } \\
\text { score }\end{array}$ & $\begin{array}{l}\text { Number of hives per } \\
24 \mathrm{~h}\end{array}$ \\
\hline 0 & None & 0 & None \\
\hline 1 & Mild (present but not annoying or troublesome) & 1 & $<20$ \\
\hline 2 & $\begin{array}{l}\text { Moderate (troublesome but does not interfere with normal daily activity } \\
\text { or sleep) }\end{array}$ & 2 & $20-50$ \\
\hline 3 & Intense (interferes with normal daily activity or sleep) & 3 & $>50$ \\
\hline
\end{tabular}

TABLE 3: UAS once-daily version - daily scoring for itch and hives

UAS: urticaria activity score

\section{Additional Information \\ Disclosures}

Human subjects: Consent was obtained or waived by all participants in this study. NHS Health Research Authority issued approval N/A. The NHS Health Research Authority has determined that this audit was not researched as defined by the UK Policy Framework for Health and Social Care Research. Animal subjects: All authors have confirmed that this study did not involve animal subjects or tissue. Conflicts of interest: In compliance with the ICMJE uniform disclosure form, all authors declare the following: Payment/services info: All authors have declared that no financial support was received from any organization for the submitted work. Financial relationships: All authors have declared that they have no financial relationships at present or within the previous three years with any organizations that might have an interest in the submitted work. Other relationships: All authors have declared that there are no other relationships or activities that could appear to have influenced the submitted work.

\section{References}

1. Kumar C, Zito PM: Omalizumab. StatPearls [Internet]. StatPearls Publishing, Treasure Island, FL; 2021.

2. Denman S, Ford K, Toolan J, Mistry A, Corps C, Wood P, Savic S: Home self-administration of omalizumab for chronic spontaneous urticaria. Br J Dermatol. 2016, 175:1405-7. 10.1111/bjd.15074

3. Modifications for adult allergy services during COVID-19 pandemic . (2020). Accessed: July 23, 2021: https://www.bsaci.org/modifications-for-adult-allergy-services-during-covid-19-pandemic/.

4. Timmermann H, Mailänder C: Home self-administration of biologics - a German survey among Omalizumab-treated patients with severe asthma and their treating physicians. Pneumologie. 2020, 74:10311. 10.1055/a-1069-0900

5. Shaker M, Briggs A, Dbouk A, Dutille E, Oppenheimer J, Greenhawt M: Estimation of health and economic benefits of clinic versus home administration of omalizumab and mepolizumab. J Allergy Clin Immunol Pract. 2020, 8:565-72. 10.1016/j.jaip.2019.09.037

6. Ghazanfar MN, Thomsen SF: Home self-administration of omalizumab. J Dermatolog Treat. 2018, 29:196. 10.1080/09546634.2017.1341611

7. Lombardi C, Bagnasco D, Passalacqua G: Biological agents for severe asthma: the evolution of the at-home self-injection approach. Curr Opin Allergy Clin Immunol. 2020, 20:421-7. 10.1097/ACI.0000000000000656

8. Menzella F, Ferrari E, Ferrucci SM, et al.: Self-administration of omalizumab: why not? A literature review and expert opinion. Expert Opin Biol Ther. 2021, 21:499-507. 10.1080/14712598.2021.1882990

9. King C, Cox F, Sloan A, McCrea P, Edgar JD, Conlon N: Rapid transition to home omalizumab treatment for chronic spontaneous urticaria during the COVID-19 pandemic: a patient perspective. World Allergy Organ J. 2021, 14:10.1016/j.waojou.2021.100587

10. Monaghesh E, Hajizadeh A: The role of telehealth during COVID-19 outbreak: a systematic review based on current evidence. BMC Public Health. 2020, 20: 10.1186/s12889-020-09301-4

11. Shenoy P, Ahmed S, Paul A, Skaria TG, Joby J, Alias B: Switching to teleconsultation for rheumatology in the wake of the COVID-19 pandemic: feasibility and patient response in India. Clin Rheumatol. 2020, 39:275762. 10.1007/s10067-020-05200-6

12. Atreya S, Kumar G, Samal J, et al.: Patients'/caregivers' perspectives on telemedicine service for advanced cancer patients during the COVID-19 pandemic: an exploratory survey. Indian J Palliat Care. 2020, 26:40-4.

13. Andrews E, Berghofer K, Long J, Prescott A, Caboral-Stevens M: Satisfaction with the use of telehealth during COVID-19: an integrative review. Int J Nurs Stud Adv. 2020, 2:10.1016/j.ijnsa.2020.100008

14. Saedon H, Gould G, Begum M, Aslam TM: Video conferencing in the intravitreal injection clinic in response to the COVID-19 pandemic. Ophthalmol Ther. 2020, 9:1-6. 10.1007/s40123-020-00262-W

15. Sharma A, Butt MI, Ajayi B, Perkins S, Umarji S, Hing C, Lui DF: A hybrid virtual fracture clinic is safe and efficacious in the COVID-19 era: stay at home and save lives. Cureus. 2021, 13:10.7759/cureus.14849

16. Popova D, Young K, Hobday D, Welman T, Pahal GS: Hand trauma virtual clinics prove effective during the COVID-19 pandemic. Hand (NY). 2021, 10.1177/15589447211017210

17. Krishna MT, Beck S, Gribbin N, et al.: The impact of COVID-19 pandemic on adult and pediatric allergy \& 


\section{Cureus}

immunology services in the UK National Health Service. J Allergy Clin Immunol Pract. 2021, 9:709-722.e2. 10.1016/j.jaip.2020.11.038 